\title{
COMMON FIXED POINTS IN HYPERBOLIC RIEMANN SURFACES AND CONVEX DOMAINS
}

\author{
MARCO ABATE AND JEAN-PIERRE VIGUÉ
}

(Communicated by Clifford J. Earle, Jr.)

\begin{abstract}
In this paper we prove that a commuting family of continuous selfmaps of a bounded convex domain in $\mathbb{C}^{n}$ which are holomorphic in the interior has a common fixed point. The proof makes use of three basic ingredients: iteration theory of holomorphic maps, a precise description of the structure of the boundary of a convex domain, and a similar result for commuting families of self-maps of a hyperbolic domain of a compact Riemann surface.
\end{abstract}

\section{INTRODUCTION}

Let $D \Subset \mathbb{C}^{n}$ be a bounded domain. In this paper we shall study families of continuous maps of $\bar{D}$ into itself that are holomorphic in $D$ and commute with each other under composition. In particular, we shall be interested in the existence of points which are fixed by any element of the family.

For the sake of brevity, let us make some definitions. Let $X$ be a topological space, and $\mathscr{F}$ a family of continuous maps of $X$ into itself (shortly, of selfmaps of $X$ ). We shall say that $\mathscr{F}$ is a commuting family if $f \circ g=g \circ f$ for every $f, g \in \mathscr{F}$. A point $x \in X$ is a fixed point of the family $\mathscr{F}$ if $f(x)=x$ for all $f \in \mathscr{F}$. Then the starting point of our paper is the following theorem, proved by Shields in 1964:

Theorem 0.1 (Shields $[\mathrm{S}])$. Let $\Delta$ be the unit disk in $\mathbb{C}$, and $\mathscr{F}$ a commuting family of continuous self-maps of $\bar{\Delta}$ holomorphic in $\Delta$. Then $\mathscr{F}$ has a fixed point in $\bar{\Delta}$.

We point out that, although every continuous self-map of $\bar{\Delta}$ has a fixed point, this result is a feature of the holomorphic structure, and not just some sort of consequence of Brouwer's theorem: indeed, there are examples of commuting continuous functions mapping the closed unit interval $[-1,1] \subset \mathbb{R}$ into itself without common fixed points (cf. Boyce [B] and Huneke [H]). See also [Co] for a detailed study of commuting holomorphic functions in $\Delta$.

Received by the editors November 29, 1989 and, in revised form, May 15, 1990.

1980 Mathematics Subject Classification (1985 Revision). Primary 32H99; Secondary 30F99, $32 \mathrm{H} 15,48 \mathrm{C} 30,52 \mathrm{~A} 20$.

The first author was partially supported by a grant from Fondazione Francesco Severi, Arezzo. 
The natural generalization of Shields' theorem to several complex variables would be an analogous statement for bounded convex domains of $\mathbb{C}^{n}$. The first step in this direction was made by Eustice [E], who in 1972 extended Shields' theorem to $\Delta^{2}=\Delta \times \Delta \subset \mathbb{C}^{2}$. Later, Suffridge [Su] found a proof valid for the euclidean unit ball $B^{n}$ of $\mathbb{C}^{n}$ (a slightly simpler argument is presented in [A4]), and Heath and Suffridge [HS] generalized the theorem to the unit polydisk $\Delta^{n}$.

Shields', Eustice's, and Suffridge's proofs were all based on the iteration theory of holomorphic maps. Recently, the iteration theory in convex domains has been thoroughly studied (see [A1, 2, 4]); this allowed the first author to generalize Shields' theorem to strongly convex domains (in [A3]). His proof was then considerably simplified by the second author and, independently, by Kuczumow and Stachura [KS], who also extended the theorem to product domains.

In this paper we shall be able to prove the complete generalization of Shields' theorem to convex domains; namely,

Theorem 0.2. Let $D$ be a bounded convex domain in $\mathbb{C}^{n}$, and $\mathscr{F}$ a commuting family of continuous self-maps of $\bar{D}$ holomorphic in $D$. Then $\mathscr{F}$ has a fixed point in $\bar{D}$.

The proof is contained in the second section of this paper; in the first section we shall extend Shields' theorem to hyperbolic domains of compact Riemann surfaces, introducing a very mild restriction on the families under consideration.

\section{Hyperbolic RiemanN SURFACES}

We begin recalling some notations and definitions. Let $X$ and $Y$ be two complex manifolds; we shall denote by $\operatorname{Hol}(X, Y)$ the space of holomorphic maps from $X$ into $Y$, endowed with the compact-open topology. Aut $(X) \subset$ $\operatorname{Hol}(X, X)$ will denote the automorphism group of $X$. A sequence $\left\{f_{k}\right\} \subset$ $\operatorname{Hol}(X, Y)$ is said to be compactly divergent if for every pair of compact sets $H \subset X$ and $K \subset Y$ we have $f_{k}(H) \cap K=\varnothing$ for $k$ sufficiently large. A family $\mathscr{F} \subset \operatorname{Hol}(X, Y)$ is said to be normal if every sequence in $\mathscr{F}$ admits a subsequence which is either compactly divergent or uniformly convergent on compact subsets.

Now let $X$ be a compact Riemann surface. A hyperbolic domain of $X$ is an open connected subset $D$ of $X$ that is hyperbolic as Riemann surface. It is known (Montel's theorem for hyperbolic Riemann surfaces; see [A4, Theorem 1.1.43, Proposition 1.1.25]) that if $X$ is any Riemann surface and $Y$ is a hyperbolic Riemann surface then the family $\operatorname{Hol}(X, Y)$ is normal. For hyperbolic domains something more is true:

Proposition 1.1. Let $D$ be a hyperbolic domain of a compact Riemann surface $X$, and $Y$ another Riemann surface. Then $\operatorname{Hol}(Y, D)$ is relatively compact in $\operatorname{Hol}(Y, X)$.

Proof. Let $D_{1} \subset X$ be a hyperbolic domain containing $D$ and such that $X \backslash D_{1}$ is finite (containing three points if $X$ is the Riemann sphere, one point if $X$ 
is a torus, even empty if $X$ is hyperbolic); it suffices to show that $\operatorname{Hol}\left(Y, D_{1}\right)$ is relatively compact in $\operatorname{Hol}(Y, X)$.

Let $\left\{f_{k}\right\}$ be a sequence in $\operatorname{Hol}\left(Y, D_{1}\right)$; we must find a subsequence converging in $\operatorname{Hol}(Y, X)$. By Montel's theorem, up to a subsequence we can assume that $\left\{f_{k}\right\}$ is compactly divergent in $\operatorname{Hol}\left(Y, D_{1}\right)$; otherwise we can extract a subsequence already converging in $\operatorname{Hol}\left(Y, D_{1}\right)$. Note that if $D_{1}=X$ the proof is finished.

Write $X \backslash D_{1}=\left\{x_{1}, \ldots, x_{p}\right\}$, and fix a compact connected subset $K_{0}$ of $Y$. We claim that there exists $x_{j_{0}} \in X \backslash D_{1}$, a subsequence $\left\{f_{k_{\nu}}\right\}$ of $\left\{f_{k}\right\}$ and a connected neighborhood $U_{0}$ of $x_{j_{0}}$ in $X$ such that for every compact connected subset $K$ of $Y$ containing $K_{0}$ and every neighborhood $U$ of $x_{j_{0}}$ in $X$ contained in $U_{0}$ we have $f_{k_{\nu}}(K) \subset U$ for all $\nu$ large enough. Since $Y$ is the increasing union of compact connected subsets containing $K_{0}$, this will imply that $f_{k_{\nu}} \rightarrow x_{j_{0}}$ in $\operatorname{Hol}(Y, X)$ as $\nu \rightarrow+\infty$, and the assertion will follow.

For $j=1, \ldots, p$ choose disjoint connected neighborhoods $U_{j}$ of $x_{j}$ in $X$. Since $\left\{f_{k}\right\}$ is compactly divergent, we have $f_{k}\left(K_{0}\right) \subset \bigcup_{j} U_{j}$ for $k$ sufficiently large. But $K_{0}$ is connected; hence there is a subsequence $\left\{f_{k_{\nu}}\right\}$ and an index $j_{0}$ such that $f_{k_{\nu}}\left(K_{0}\right) \subset U_{j_{0}}=U_{0}$ for all $\nu \in \mathbb{N}$.

Now let $K$ be any compact connected subset of $Y$ containing $K_{0}$, and $U$ any neighborhood of $x_{j_{0}}$ in $X$ contained in $U_{0}$. Since $\left\{f_{k_{\nu}}\right\}$ is still compactly divergent, we have

$$
f_{k_{\nu}}(K) \subset U \cup \bigcup_{j \neq j_{0}} U_{j}
$$

for $\nu$ large enough. But $K \supset K_{0}$ is connected, and $f_{k_{\nu}}(K) \cap U_{0} \neq \varnothing$ for every $\nu \in \mathbb{N}$; therefore, $f_{k_{\nu}}(K) \subset U$ for $\nu$ sufficiently large, and we are done. Q.E.D.

This proposition can be expressed by saying that $D$ is hyperbolically imbedded in $X$. It is worth remarking that Kiernan $[\mathrm{K}]$ has shown that this is equivalent to the following geometrical fact: if $\left\{z_{\nu}\right\}$ and $\left\{w_{\nu}\right\}$ are sequences in $D$ such that $z_{\nu} \rightarrow z \in \bar{D}, w_{\nu} \rightarrow w \in \bar{D}$, and $k_{D}\left(z_{\nu}, w_{\nu}\right) \rightarrow 0$ as $\nu \rightarrow+\infty$, where $k_{D}$ is the Poincare distance on $D$, then $z=w$.

As already anticipated in the introduction, theorems on common fixed points are often proved using iteration theory. In our case, we shall need only a few easy results, that we are now going to describe.

Let $X$ be a complex manifold; we shall denote by id $_{X}$ the identity map of $X$. Take a map $f \in \operatorname{Hol}(X, X)$; the $k$ th iterate $f^{k}$ of $f$ is inductively defined by $f^{0}=\operatorname{id}_{X}$ and $f^{k}=f \circ f^{k-1}$. A limit point of the sequence $\left\{f^{k}\right\}$ is the limit of a subsequence $\left\{f^{k_{\nu}}\right\}$ such that $k_{\nu} \rightarrow+\infty$ as $\nu \rightarrow+\infty$.

Our first goal is a precise description of the limit points of a sequence of iterates. To obtain it, we need two preliminary lemmas. The first one is of 
topological character:

Lemma 1.2. Let $X, Y$ be two locally compact locally connected Hausdorff spaces, and $\left\{\varphi_{\nu}\right\}$ a sequence of continuous open maps of $X$ into $Y$, converging for the compact-open topology to a continuous map $\varphi: X \rightarrow Y$. Suppose that $z_{0} \in X$ is an isolated point of $\varphi^{-1}\left(\varphi\left(z_{0}\right)\right)$. Then for any neighborhood $U$ of $z_{0}$ there is a $\nu_{0} \in \mathbb{N}$ such that

$$
\varphi\left(z_{0}\right) \in \varphi_{\nu}(U) \quad \forall \nu \geq \nu_{0} .
$$

Proof. See [A4, Lemma 2.1.19] or [N, Proposition 5, p. 79]. Q.E.D.

The second lemma holds in a general setting too:

Lemma 1.3. Let $X$ be a complex manifold, and $f \in \operatorname{Hol}(X, X)$. Then an automorphism of $X$ can be a limit of $\left\{f^{k}\right\}$ only if $f$ itself is an automorphism of $X$.

Proof. Assume $g \in \operatorname{Aut}(X)$ be a limit point of $\left\{f^{k}\right\}$. Clearly, $f$ is one-to-one; in particular, it is an open map. Let $\left\{f^{k_{\nu}}\right\}$ be a subsequence converging to $g$, and take $z_{0} \in X$. Then Lemma 1.2 applied to the sequence $\varphi_{\nu}=f^{k_{\nu}}$ and to the point $g^{-1}\left(z_{0}\right)$ shows that $z_{0}=g\left(g^{-1}\left(z_{0}\right)\right) \in f(X)$. Being $z_{0}$ arbitrary, this implies that $f$ is onto, and we are done. Q.E.D.

It should be remarked that if $X$ actually is a Riemann surface, then it is possible to prove this lemma using the classical Hurwitz theorem for Riemann surfaces [A4, Corollary 1.1.36] instead of Lemma 1.2.

And now we can completely describe the limit points of a sequence of iterates:

Theorem 1.4. Let $D$ be a hyperbolic domain of a compact Riemann surface $X$, and $f \in \operatorname{Hol}(D, D)$. Let $h: D \rightarrow X$ be a limit point in $\operatorname{Hol}(D, X)$ of the sequence $\left\{f^{k}\right\}$. Then either

(i) $h$ is a constant $z_{0} \in \bar{D}$, or

(ii) $h$ is an automorphism of $D$. In this case, $f$ is an automorphism too.

Proof. Clearly, $h(D) \subset \bar{D}$. If $h(D) \cap \partial D \neq \varnothing$, by the open mapping theorem $h$ is constant, and we are in case (i); therefore, we can assume $h \in \operatorname{Hol}(D, D)$.

Choose a subsequence $\left\{f^{k_{\nu}}\right\}$ converging to $h$; we can assume that $m_{\nu}=$ $k_{\nu+1}-k_{\nu}$ tends to infinity as $\nu \rightarrow+\infty$. By Montel's theorem, up to a subsequence we can also suppose that $\left\{f^{m_{\nu}}\right\}$ either converges to a holomorphic map $g$ or is compactly divergent.

Suppose $h$ is not constant; we must prove that both $f$ and $h$ are automorphisms of $D$. For any $z \in D$ we have

$$
\lim _{\nu \rightarrow \infty} f^{m_{\nu}}\left(f^{k_{\nu}}(z)\right)=\lim _{\nu \rightarrow \infty} f^{k_{\nu+1}}(z)=h(z) ;
$$

therefore, $\left\{f^{m_{\nu}}\right\}$ cannot be compactly divergent, and $g$ is the identity on the open subset $h(D)$ of $D$. Hence $g=\mathrm{id}_{D}$; by Lemma 1.3, $f$ is an automorphism. 
It remains to show that $h$ itself is an automorphism. Set $f_{\nu}=f^{k_{\nu}}$ and $g_{\nu}=f_{\nu}^{-1}$. By Montel's theorem, up to a subsequence we can assume that either $g_{\nu} \rightarrow g \in \operatorname{Hol}(D, D)$ or $\left\{g_{\nu}\right\}$ is compactly divergent. Since for any $z \in D$ we have $f_{\nu}(z) \rightarrow h(z) \in D$ and $g_{\nu}\left(f_{\nu}(z)\right)=z$, the sequence $\left\{g_{\nu}\right\}$ cannot be compactly divergent. Then

$$
g(h(z))=\lim _{\nu \rightarrow \infty} g_{\nu}\left(f_{\nu}(z)\right)=z \quad \forall z \in D,
$$

and, since $g(D) \subset D$,

$$
h(g(z))=\lim _{\nu \rightarrow \infty} f_{\nu}\left(g_{\nu}(z)\right)=z \quad \forall z \in D ;
$$

therefore $g=h^{-1}$, and $h$ is an automorphism. Q.E.D.

We are now able to prove the announced generalization of Shields' theorem to hyperbolic domains:

Theorem 1.5. Let $D$ be a hyperbolic domain of a compact Riemann surface $X$, and $\mathscr{F}$ a commuting family of continuous self-maps of $\bar{D}$ holomorphic in $D$. Assume either:

(i) D is simply connected, or

(ii) $D$ is multiply connected and $\mathscr{F}$ is not contained in $\operatorname{Aut}(D)$.

Then $\mathscr{F}$ has a fixed point.

Proof. We begin remarking that if $\mathscr{F}$ contains a constant map $z_{0} \in \bar{D}$, then $z_{0}$ is clearly a fixed point of $\mathscr{F}$; hence we shall assume that $\mathscr{F}$ does not contain constant maps. By the open map theorem, then, every element of $\mathscr{F}$ sends $D$ into itself.

Suppose, for the moment, that $\mathscr{F}$ is not contained in $\operatorname{Aut}(D)$, and take $f_{0} \in \mathscr{F} \backslash \operatorname{Aut}(D)$. Let $h$ be a limit point in $\operatorname{Hol}(D, X)$ of the sequence of iterates of $f_{0} ; h$ exists by Proposition 1.1, and it is a constant $z_{0} \in \bar{D}$ by Theorem 1.4. Choose a subsequence $\left\{f_{0}^{k_{\nu}}\right\}$ converging to $h$. Then for every $g \in \mathscr{F}$ we have

$$
g\left(z_{0}\right)=\lim _{\nu \rightarrow \infty} g\left(f_{0}^{k_{\nu}}(z)\right)=\lim _{\nu \rightarrow \infty} f_{0}^{k_{\nu}}(g(z))=z_{0},
$$

where $z$ is any point of $D$; therefore, $z_{0}$ is a fixed point of $\mathscr{F}$.

It remains to study the case $\mathscr{F} \subset \operatorname{Aut}(D)$ and $D$ simply connected; in particular, $D$ is biholomorphic to the upper half-plane $H^{+}$of $\mathbb{C}$. Take $f_{0} \in \mathscr{F}$. If $f_{0}$ has no fixed points in $D$, using the explicit expression of automorphisms of $\mathrm{H}^{+}$it is easy to see that the sequence $\left\{f_{0}^{k}\right\}$ is compactly divergent. Then (by Proposition 1.1 and Theorem 1.4) there is a subsequence $\left\{f_{0}^{k_{\nu}}\right\}$ converging toward a constant $z_{0} \in \partial D$, and we find as before that $z_{0}$ is a fixed point of $\mathscr{F}$.

Finally, assume every $f \in \mathscr{F}$ has a fixed point in $D$. Take $f_{0} \in \mathscr{F}$. Clearly, we can assume $f_{0} \neq \mathrm{id}_{D}$; so $f_{0}$ has a unique fixed point $z_{0} \in D$. Now, for 
every $f \in \mathscr{F}$ we have

$$
f_{0}\left(f\left(z_{0}\right)\right)=f\left(f_{0}\left(z_{0}\right)\right)=f\left(z_{0}\right)
$$

therefore, $f\left(z_{0}\right)=z_{0}$, and we are done. Q.E.D.

Two remarks are in order. First of all, in multiply-connected domains there actually exist commuting families of automorphisms without fixed points; indeed, there are automorphisms (continuous up to the boundary and) without fixed points. Take for instance

$$
D=\{z \in \mathbb{C}|1<| z \mid<2\}
$$

and $f(z)=-z$. Second, Theorem 1.5 is more general then [A4, Theorem 1.3.22], and its proof requires considerably less technical tools; in particular, it is not necessary to study the boundary behavior of the universal covering map.

\section{Convex domains}

This section is devoted to the proof of Theorem 0.2. The main tool (besides some results on iteration theory and fixed point sets in convex domains to be recalled later) is a description of the structure of the boundary of a convex domain. We begin with a (very easy) sort of maximum principle:

Lemma 2.1. Let $D$ be a bounded convex domain in $\mathbb{C}^{n}, X$ a (connected) complex manifold, and $f \in \operatorname{Hol}\left(X, \mathbb{C}^{n}\right)$ such that $f(X) \subset \bar{D}$. Then either $f(X) \subset D$ or $f(X) \subset \partial D$.

Proof. Assume there exists $w_{0} \in X$ such that $x_{0}=f\left(w_{0}\right) \in \partial D$; we must show that $f(X) \subset \partial D$. By the Hahn-Banach theorem, we can find a $\mathbb{C}$-linear map $\sigma: \mathbb{C}^{n} \rightarrow \mathbb{C}$ such that

$$
\operatorname{Re} \sigma(z)<\operatorname{Re} \sigma\left(x_{0}\right) \quad \forall z \in D .
$$

Therefore $\operatorname{Re} \sigma \circ f \leq \operatorname{Re} \sigma \circ f\left(w_{0}\right)$ on $X$; the maximum principle then implies $\sigma \circ f \equiv \sigma \circ f\left(w_{0}\right)$, and in particular $f(X) \subset \partial D$. Q.E.D.

Using a more refined version of the previous argument, we can associate with every point $x_{0}$ in the boundary of a bounded convex domain $D \Subset \mathbb{C}^{n}$, a convex domain of smaller dimension which is, in a certain sense, the natural range of every holomorphic map $f: X \rightarrow \bar{D}$ such that $x_{0} \in f(X)$ More precisely, we have

Proposition 2.2. Let $D$ be a bounded convex domain in $\mathbb{C}^{n}$, and take $x_{0} \in \partial D$. Then there exists a complex affine subspace $L$ of $\mathbb{C}^{n}$ satisfying the following conditions:

(i) $D \cap L=\varnothing$;

(ii) $\bar{D} \cap L$ is the closure of a bounded convex domain $D_{0}$ of $L$;

(iii) $x_{0} \in D_{0}$; 
(iv) for every connected complex manifold $X$ and holomorphic map $f: X \rightarrow$ $\mathbb{C}^{n}$ such that $f(X) \subset \bar{D}$ and $f(X) \cap \bar{D}_{0} \neq \varnothing$ we have $f(X) \subset \overline{D_{0}}=$ $\bar{D} \cap L$;

(v) for every connected complex manifold $X$ and holomorphic map $f: X \rightarrow$ $\mathbb{C}^{n}$ such that $f(X) \subset \bar{D}$ and $x_{0} \in f(X)$ we have $f(X) \subset D_{0}$.

Proof. We shall prove by induction on $n$ the existence of $L$ and $D_{0}$ satisfying (i)-(iv); (v) will then follow from (iii), (iv), and Lemma 2.1.

For $n=1$, by the open mapping theorem we can take $L=D_{0}=\left\{x_{0}\right\}$; so assume $n>1$. Without loss of generality, we can suppose that $x_{0}$ is the origin. By the Hahn-Banach theorem, there exists a $\mathbb{C}$-linear map $\sigma_{1}: \mathbb{C}^{n} \rightarrow \mathbb{C}$ such that $\operatorname{Re} \sigma_{1}<0$ on $D$; set $V_{1}=\operatorname{ker} \sigma_{1}$. Let $W_{1}$ be the real subspace of $V_{1}$ generated by $\bar{D} \cap V_{1}$, and set $L_{1}=W_{1} \cap i W_{1}$. Since $\bar{D} \cap V_{1}$ is convex and generates $W_{1}$, there is a convex open subset $D_{1}^{\prime}$ of $W_{1}$ such that $\bar{D} \cap V_{1}=\overline{D_{1}^{\prime}}$; set $D_{1}=D_{1}^{\prime} \cap L_{1}$. Clearly, $x_{0}=0 \in \overline{D_{1}}=\bar{D} \cap L_{1}$. Note that the complex dimension of $L_{1}$ is strictly less than $n$.

Let $f \in \operatorname{Hol}\left(X, \mathbb{C}^{n}\right)$ be such that $f(X) \subset \bar{D}$ and $f(X) \cap \overline{D_{1}} \neq \varnothing$; fix $w_{0} \in f^{-1}\left(\overline{D_{1}}\right)$. Since $\operatorname{Re} \sigma_{1} \circ f \leq 0$ on $X$ and $\sigma_{1} \circ f\left(w_{0}\right)=0$, the maximum principle implies $\sigma_{1} \circ f \equiv 0$, i.e., $f(X) \subset \bar{D} \cap V_{1}$. In particular, $f$ is a holomorphic map from $X$ to $V_{1}$.

Now, there exists a (possibly empty) set $\left\{\tau_{1}, \ldots, \tau_{r}\right\}$ of $\mathbb{C}$-linear forms on $V_{1}$ such that

$$
W_{1}=\bigcap_{j=1}^{r} \operatorname{ker} \operatorname{Re} \tau_{j} \quad \text { and } \quad L_{1}=\bigcap_{j=1}^{r} \operatorname{ker} \tau_{j} .
$$

Since $\bar{D} \cap V_{1}$ is contained in $W_{1}$, we have $\operatorname{Re} \tau_{j} \circ f \equiv 0$ on $X$. Being $\tau_{j} \circ$ $f\left(w_{0}\right)=0$, this yields $\tau_{j} \circ f \equiv 0$ on $X$ for every $j=1, \ldots, r$, that is $f(X) \subset \bar{D} \cap L_{1}=\overline{D_{1}}$.

In other words we have constructed a complex subspace $L_{1}$ and a bounded convex domain $D_{1}$ of $L_{1}$ satisfying (i), (ii) and (iv). If $x_{0} \in D_{1}$ we are done; otherwise, $x_{0} \in \partial D_{1}$ and we can apply the induction hypothesis. Q.E.D.

We shall use this proposition to build up an induction argument; but before the proof of Theorem 0.2 we recall another few facts on convex domains. First of all, we shall make use of the following result of iteration theory:

Theorem 2.3 [A2; A4, Theorems 2.4.3, 2.4.20; KS]. Let $D$ be a bounded convex domain of $\mathbb{C}^{n}$, and $f \in \operatorname{Hol}(D, D)$. Then the following facts are equivalent:

(i) $f$ has a fixed point in $D$;

(ii) the sequence of iterates of $f$ is not compactly divergent;

(iii) the sequence of iterates of $f$ is relatively compact in $\operatorname{Hol}(D, D)$;

(iv) for every point $z \in D$ the sequence $\left\{f^{k}(z)\right\}$ is relatively compact in $D$;

(v) for one point $z_{0} \in D$ the sequence $\left\{f^{k}\left(z_{0}\right)\right\}$ is relatively compact in $D$. 
It should be remarked that the convexity of $D$ is required only to prove that (v) $\Rightarrow$ (i); the equivalence of (ii), (iii), (iv), and (v) holds for any taut manifold, and (i) $\Rightarrow$ (ii) is obvious.

To state the second result we recall a couple of definitions. Let $X$ be a complex manifold; a holomorphic retraction of $X$ is a map $\rho \in \operatorname{Hol}(X, X)$ such that $\rho^{2}=\rho$; its image is a holomorphic retract of $X$. It should be noticed that a holomorphic retract is always a smooth submanifold [R, C]. In convex domains, fixed point sets and holomorphic retracts are one and the same thing:

Theorem 2.4 [V1, 2]. Let $D$ be a bounded convex domain of $\mathbb{C}^{n}$, and $f \in$ $\operatorname{Hol}(D, D)$. Let $F$ be the set of fixed points of $f$ in $D$, and assume $F \neq \varnothing$. Then there exists a holomorphic retraction $\rho_{f}: D \rightarrow D$ such that $\rho_{f}(D)=F$. In particular, $F$ is a closed connected submanifold of $D$.

We are almost ready to prove Theorem 0.2 . We only need another observation:

Lemma 2.5. Let $D$ be a bounded convex domain of $\mathbb{C}^{n}$, and let $L$ be a complex affine subspace of $\mathbb{C}^{n}$ such that $L \cap D=\varnothing$ and $L \cap \partial D \neq \varnothing$. Let $D_{1}$ be the interior of $L \cap \partial D$ in $L$. Then for every continuous function $f: \bar{D} \rightarrow \mathbb{C}^{m}$ which is holomorphic in $D$ the restriction of $f$ to $D_{1}$ is still holomorphic.

Proof. Without loss of generality we can assume $0 \in D$. For every $k \in \mathbb{N}^{*}$ define $f_{k}: D_{1} \rightarrow \mathbb{C}^{m}$ by

$$
f_{k}(z)=f((1-1 / k) z) .
$$

It is clear that every $f_{k}$ is holomorphic; since $\left.f_{k} \rightarrow f\right|_{D_{1}}$ uniformly, the assertion follows. Q.E.D.

And finally:

Theorem 0.2. Let $D$ be a bounded convex domain in $\mathbb{C}^{n}$, and $\mathscr{F}$ a commuting family of continuous self-maps of $\bar{D}$ holomorphic in $D$. Then $\mathscr{F}$ has a fixed point in $\bar{D}$.

Proof. By induction on $n$. For $n=1$ it is just Theorem 1.5; so assume $n>1$. We must examine three mutually exclusive (by Lemma 2.1) cases:

(a) Every $f \in \mathscr{F}$ has a fixed point in $D$. For every $f \in \operatorname{Hol}(D, D)$ let Fix $(f)$ denote the fixed point set of $f$ in $D$. Take $f, g \in \mathscr{F}$; since $f$ and $g$ commute, we have $g(\operatorname{Fix}(f)) \subset \operatorname{Fix}(f)$. Let $\rho_{f}: D \rightarrow \operatorname{Fix}(f)$ be the holomorphic retraction given by Theorem 2.4, and set $\tilde{g}=\rho_{f} \circ g \in \operatorname{Hol}(D, D)$. For every $z \in \operatorname{Fix}(f)$ and $k \in \mathbb{N}$ we have $\tilde{g}^{k}(z)=g^{k}(z)$; hence, by Theorem 2.3, $\operatorname{Fix}(\tilde{g}) \neq \varnothing$. Since $\operatorname{Fix}(\tilde{g})=\operatorname{Fix}(f) \cap \operatorname{Fix}(g)$, it follows from Theorem 2.4 that $\operatorname{Fix}(f) \cap \operatorname{Fix}(g)$ is a holomorphic retract of $D$.

Using an induction argument it is now easy to show that $\operatorname{Fix}\left(f_{1}\right) \cap \cdots \cap \operatorname{Fix}\left(f_{r}\right)$ is a holomorphic retract (and so a nonempty closed smooth submanifold) of $D$ for every $r \in \mathbb{N}$ and $f_{1}, \ldots, f_{r} \in \mathscr{F}$. Set

$$
d=\min \left\{\operatorname{dim}\left[\operatorname{Fix}\left(f_{1}\right) \cap \cdots \cap \operatorname{Fix}\left(f_{r}\right)\right] \mid r \in \mathbb{N}, f_{1}, \ldots, f_{r} \in \mathscr{F}\right\} \geq 0,
$$


and pick $f_{1}, \ldots, f_{r_{0}} \in \mathscr{F}$ so that the dimension of $F=\operatorname{Fix}\left(f_{1}\right) \cap \cdots \cap \operatorname{Fix}\left(f_{r_{0}}\right)$ is exactly $d$. This implies that $F \cap \operatorname{Fix}(f)$ is (a closed connected submanifold of equal dimension and hence) equal to $F$ for every $f \in \mathscr{F}$, and so every point in $F$ is fixed by $\mathscr{F}$.

(b) There exists $f_{0} \in \mathscr{F}$ such that $f_{0}(D) \subset \partial D$; in particular, $f_{0}$ has no fixed points in $D$. Fix $z_{0} \in D$ and let $x_{0}=f\left(z_{0}\right) \in \partial D$. Using Proposition 2.2 we associate to $x_{0}$ a complex affine subspace $L$ of $\mathbb{C}^{n}$ and a bounded convex domain $D_{0}$ of $L$ whose closure is $\partial D \cap L$ such that $x_{0} \in f_{0}(D) \subset D_{0}$; clearly, $f_{0}(\bar{D}) \subset \overline{D_{0}}$.

Take $g \in \mathscr{F}$. By Lemma 2.5, $\left.g\right|_{D_{0}}: D_{0} \rightarrow \mathbb{C}^{n}$ is holomorphic. Now $g\left(x_{0}\right)=$ $f\left(g\left(z_{0}\right)\right) \in \overline{D_{0}}$; hence, by Proposition 2.2 (iv), $g\left(\overline{D_{0}}\right) \subset \overline{D_{0}}$. Therefore

$$
\left.\mathscr{F}\right|_{D_{0}}=\left\{\left.g\right|_{D_{0}} \mid g \in \mathscr{F}\right\}
$$

is a commuting family of continuous self-maps of $\overline{D_{0}}$ holomorphic in $D_{0}$, and we can conclude by applying the induction hypothesis.

(c) We have $f(D) \subset D$ for every $f \in \mathscr{F}$ and there is $f_{0} \in \mathscr{F}$ without fixed points in $D$. By Theorem 2.3, the sequence of iterates of $f_{0}$ is compactly divergent; therefore, if $h \in \operatorname{Hol}\left(D, \mathbb{C}^{n}\right)$ is a limit point of $\left\{f_{0}^{k}\right\}$ (such an $h$ exists because $D$ is bounded) we have $h(D) \subset \partial D$. Furthermore,

$$
f(h(z))=h(f(z)) \quad \forall f \in \mathscr{F}, \forall z \in D,
$$

because every $f \in \mathscr{F}$ commutes with $f_{0}$ and sends $D$ into itself. Therefore we can repeat the argument used in case (b), replacing $f_{0}$ by $h$, and we are done. Q.E.D.

\section{REFERENCES}

[A1] M. Abate, Horospheres and iterates of holomorphic maps, Math. Z. 198 (1988), 225-238.

[A2] - Iteration theory on weakly convex domains, Seminar in Complex Analysis and Geometry 1988, Editel, Rende, Cosenza, 1990.

[A3] _ Common fixed points of commuting holomorphic maps, Math. Ann. 283 (1989), 645655.

[A4] _ Iteration theory of holomorphic maps on taut manifolds, Mediterranean Press, Rende, Cosenza, 1989.

[B] W. M. Boyce, Commuting functions with no common fixed points, Trans. Amer. Math. Soc. 137 (1969), 77-92.

[C] H. Cartan, Sur les rétractions d'une variété, C. R. Acad. Sci. Paris 303 (1986), 715-716.

[Co] C. C. Cowen, Commuting analytic functions, Trans. Amer. Math. Soc. 283 (1984), 685-695.

[E] D. J. Eustice, Holomorphic idempotents and common fixed points on the 2-disk, Michigan Math. J. 19 (1972), 347-352.

[HS] L. F. Heath and T. J. Suffridge, Holomorphic retracts in complex n-space, Illinois J. Math. 25 (1981), 125-135.

[H] J. P. Huneke, On common fixed points of commuting continuous functions on an interval, Trans. Amer. Math. Soc. 139 (1969), 371-381.

[K] P. Kiernan, Hyperbolically imbedded spaces and the big Picard theorem, Math. Ann. 204 (1973), 203-209. 
[KS] T. Kuczumow and A. Stachura, Iterates of holomorphic and $k_{D}$-nonexpansive mappings in convex domains in $\mathbb{C}^{n}$, Adv. in Math. 81 (1990), 90-98.

[N] R. Narasimhan, Several complex variables, Univ. of Chicago Press, Chicago, 1971.

[R] H. Rossi, Vector fields on analytic spaces, Ann. of Math. 78 (1963), 455-467.

[S] A. L. Shields, On fixed points of commuting analytic functions, Proc. Amer. Math. Soc. 15 (1964), 703-706.

[Su] T. J. Suffridge, Common fixed points of commuting holomorphic maps of the hyperball, Michigan Math. J. 21 (1974), 309-314.

[V1] J.-P. Vigué, Géodésiques complexes et points fixes d'applications holomorphes, Adv. in Math. 52 (1984), 241-247.

[V2] _ Points fixes d'applications holomorphes dans un domaine borné convexe de $\mathbb{C}^{n}$, Trans. Amer. Math. Soc. 289 (1985), 345-353.

Dipartimento di Matematica, Seconda Università di Roma, Via O. Raimondo, 00133 ROMA, ITALY

Département de Mathématiques, Université de Poitiers, 40 Avenue du Recteur Pineau, 86022 PoITIERS, France 